Jean-Pierre Bouvet

\section{ADRESSE}

J.P. Bouvet : Inserm U. 430, Hôpital Broussais, 96, rue Didot, 75674 Paris Cedex 14, France.

\title{
Complexité de la première barrière de défense immunitaire dans les muqueuses
}

L'immunité humorale des muqueuses dépend de plusieurs mécanismes complémentaires apparus par étapes successives au cours de l'évolution. Il est maintenant établi que les immunoglobulines A sécrétoires (S-IgA) ne constituent pas le seul isotype d'immunoglobuline présent dans les sécrétions et que leur nature n'est pas univoque. Ces molécules sont essentiellement des anticorps naturels polyréactifs, qui existent avant tout contact avec l'agent pathogène et forment l'essentiel de la première barrière de défense au niveau des muqueuses. Les S-IgA peuvent également être induites par contact avec un antigène étranger, mais cette induction est inefficace, ce qui explique les difficultés de mise au point des vaccinations par voie muqueuse. La mise en évidence de fragments Fab de haute affinité issus du catabolisme hépatique des IgG sériques dans le tube digestif, et d'une régionalisation des anticorps de type IgG dans les muqueuses ont renouvelé les concepts classiques de l'immunité humorale. Ces résultats récents ouvrent de nouveaux champs d'investigation pour les vaccinations.

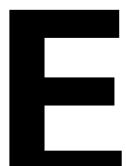

$\mathrm{n}$ dehors de la voie transcutanée, les agents pathogènes pénètrent aussi dans l'organisme par voie muqueuse. Même si l'on fait exception de l'immense surface alvéolaire, les muqueuses correspondent à une surface d'échange de plus de $400 \mathrm{~m}^{2}$, généralement de type monocouche, au contact d'une flore microbienne dont les constituants sont 100 fois plus nombreux que les cellules eucaryotes de l'organisme. Plus de $70 \%$ des cellules immunitaires et des anticorps sont mobilisés en permanence pour résister à la pénétration de ces germes, qu'ils soient potentiellement (commensaux) ou régulièrement pathogènes. Les processus de défense des muqueuses sont probablement apparus par vagues successives, et font appel à des mécanismes variés, additifs et/ou complémentaires [1]. Leur rôle essentiel est de limiter l'importance de l'inoculum microbien qui traverse la muqueuse, facteur-clé dans le déclenchement de la maladie et sa sévérité. Le rôle probablement important des lymphocytes intra-épithéliaux [2] ne sera pas 
abordé dans cet exposé, qui sera focalisé sur les défenses relayées par les anticorps des sécrétions. La plupart des études actuelles portent essentiellement sur les mécanismes de transcytose des IgA sécrétoires (SIgA), largement majoritaires dans le lait et la salive, ainsi que sur les mécanismes de la réponse induite au niveau des plaques de Peyer. Cependant, des travaux récents ont permis d'élargir ce champ d'investigation dont l'intérêt est le développement possible de vaccinations muqueuses.

\section{Les outils: les immunoglobulines des sécrétions}

Les immunoglobulines S-IgA sont prédominantes dans les sécrétions humaines les plus facilement accessibles. Leur structure originale leur procure une résistance très élevée à la dégradation par les protéases endogènes (figure 1). Les $\mathrm{S}-\mathrm{IgA}$ sont des molécules polymériques (généralement des dimères), comportant une chaîne de jonction appelée chaîne J. Grâce à cette chaîne enfouie dans la molécule, les IgA acquièrent une conformation qui leur permet de se fixer sur des structures membranaires, les récepteurs poly-Ig, situés au pôle baso-latéral de la cellule épithéliale. Les complexes IgA-récepteurs sont transportés de façon active vers le pôle apical de la cellule épithéliale, d'où ils sont libérés dans la lumière épithélio-glandulaire après clivage enzymatique de leurs attaches membranaires. Le résultat de cette transcytose est la libération de molécules dont la région «fragile» $(\mathrm{Fc} \alpha)$ est protégée contre la dégradation protéolytique par la fixation covalente des fragments extracellulaires des récepteurs, appelés "composants sécrétoires » $(\mathrm{SC})$. Le caractère polymérique des S-IgA augmente leur affinité fonctionnelle, tandis que le remplacement de la région charnière (telle qu'elle existe dans les IgG) par une pseudo-charnière courte et glycosylée (IgA1), ou rudimentaire (IgA2), complète leur résistance aux protéases endogènes.

Il existe également d'autres isotypes d'anticorps dans les sécrétions. En effet, les IgM peuvent aussi être sécrétées par transcytose sous forme de SIgM, très peu abondantes et très sensibles aux protéases malgré la présence de composants sécrétoires. Les IgG sont largement majoritaires dans les sécrétions génitales [3], et sont abondantes dans la lumière intestinale, sous forme de fragments Fab. A

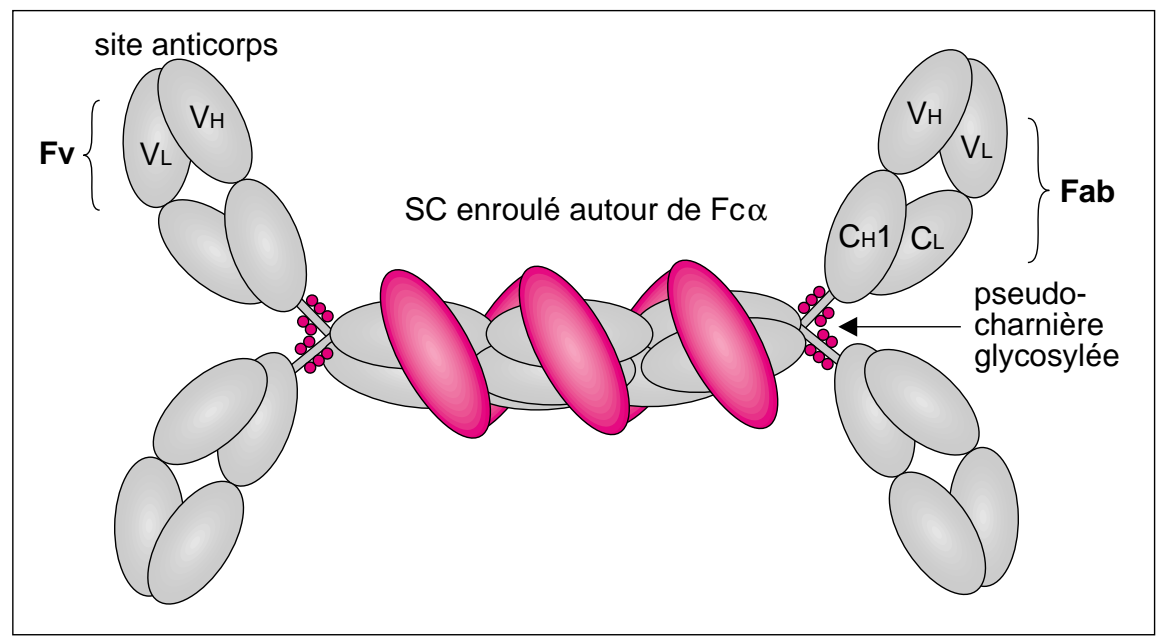

Figure 1. Structure d'une S-IgA1. La molécule d'lgA (domaines clairs) est dimérique. Cette structure lui confère 4 sites anticorps situés sur les fragments $\mathrm{Fab}(\mathrm{VH}+\mathrm{VL}+\mathrm{CH} 1+\mathrm{CL})$ et augmente ainsi son affinité fonctionnelle d'un facteur 100. La région charnière présente sur les IgG est remplacée par une pseudo-charnière o-glycosylée, mieux protégée contre l'action de nombreuses enzymes, à l'exception des lgA1-protéases. Le composant sécrétoire (SC, domaines roses) emmaillote le fragment Fco, rendant la molécule résistante aux protéases endogènes. La chaîne $\mathrm{J}$, indispensable à cette liaison, est inapparente. La liaison avec la protéine Fv a lieu sur le fragment Fv (VH + VL) la naissance, les fragments Fab $\gamma$ du méconium sont d'origine maternelle. Ils constituent le seul agent de défense de l'intestin pendant la première semaine postnatale [4]. Les IgE sont relativement abondantes à l'intérieur de la muqueuse où elles se fixent sur les nombreux mastocytes locaux. Des plasmocytes à IgD ont été observés dans la muqueuse nasale dans les cas de déficit en IgA, et cette augmentation serait de mauvais pronostic.

\section{Les mécanismes d'action: exclusion et élimination immunes}

Décrite par Stokes [5], «l'exclusion immune" constitue l'activité de base des anticorps présents dans les sécrétions (figure 2). La fixation de l'anticorps sur l'antigène dans la lumière sécrétoire, peut entraîner l'agglutination de l'agent pathogène, augmentant ainsi son élimination par le flux du mucus. Elle peut également neutraliser les toxines pathogènes et inhiber les adressines microbiennes qui permettent l'adhérence à la cellule épithéliale. Les anticorps S-IgA du lait maternel ainsi que, fréquemment, les IgG agissent par ce mécanisme.

«L'élimination immune », décrite plus récemment [6, 7], est associée à la transcytose, au cours de laquelle des complexes immuns du stroma sousépithélial, ou des virus intra-épithéliaux, sont transportés vers la lumière muqueuse par les IgA ou les IgM polymériques spécifiques de l'agent pathogène. La transcytose permet ainsi de «nettoyer» la muqueuse en relarguant l'agent pathogène dans les sécrétions où il sera éliminé par le flux du mucus. La transcytose des complexes immuns peut être facilitée par la présence d'anticorps IgG neutralisants fixés à l'agent pathogène.

Les IgE augmenteraient la perméabilité de la muqueuse et faciliteraient ainsi la diffusion des anticorps de la muqueuse vers les sécrétions. Elles auraient également une activité antiparasitaire [8].

\section{Anticorps induits et anticorps naturels}

Dans un article publié en 1927, Besredka attribuait les anticorps des sécrétions à un système immunitaire particulier [9]. La vision moderne de 


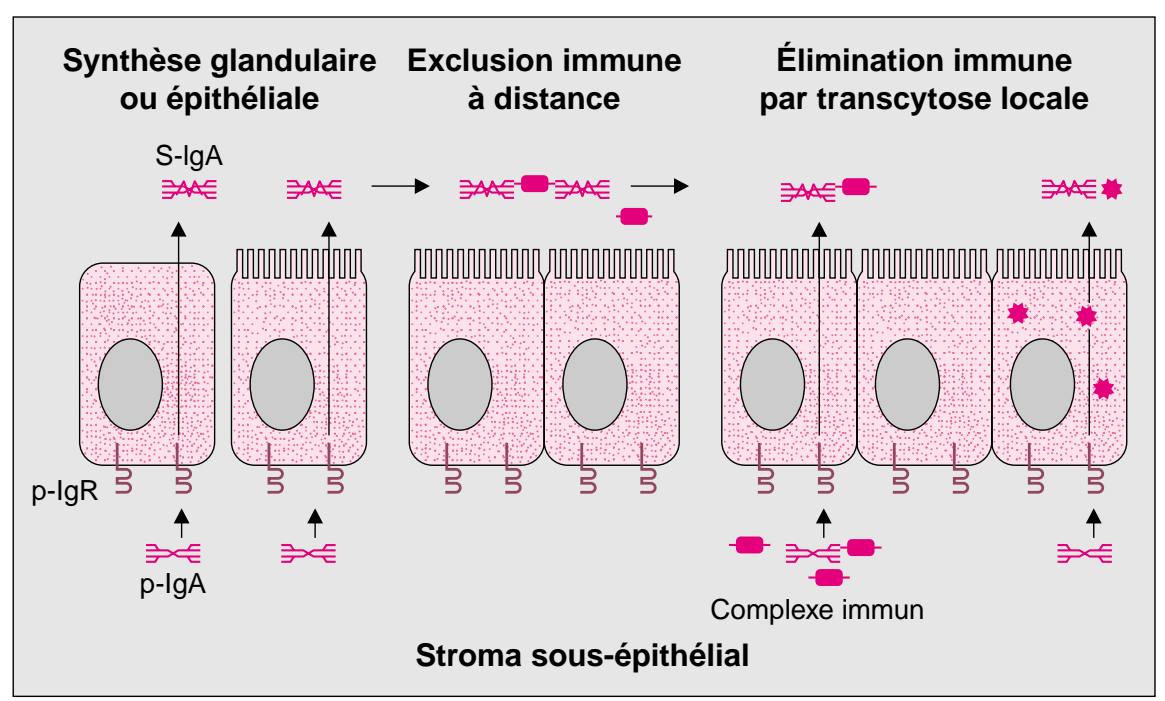

Figure 2. Synthèse et rôle des S-IgA. Les IgA polymères ( $p$-lgA) sont synthétisées par les plasmocytes du stroma sous-épithélial des muqueuses (cellules avec bordure en brosse) et des glandes exocrines ou des épithéliums canalaires (cellule sans bordure en brosse). Elles se fixent (flèches verticales) sur le récepteur des immunoglobulines polymériques ( $p$-lgR $=S C$ membranaire) et sont transportées de façon active (transcytose) vers le pôle apical (endoluminal) de la cellule d'où elles sont libérées par clivage protéolytique de l'attache membranaire du SC. Elles sont entraînées ensuite par l'écoulement des sécrétions (flèches horizontales) où elles peuvent neutraliser ou agglutiner les agents pathogènes (ici des bactéries en rouge). Cette exclusion immune peut avoir lieu à distance du site de synthèse, notamment dans le tube digestif. À l'inverse, dans l'élimination immune, les anticorps IgA synthétisés sur place forment des complexes immuns avec les agents pathogènes présents dans le stroma et peuvent les entraîner dans la lumière muqueuse durant la transcytose. Par le même mécanisme de transcytose ces anticorps peuvent capturer et éliminer des virus intracellulaires.

ce système, récemment synthétisée par Brandtzaeg [10], comporte des tissus lymphoïdes différenciés présents dans différentes muqueuses (mucosa-associated lymphoid tissues, MALT), dont le type est la plaque de Peyer intestinale. Ces tissus sont stimulés par des antigènes traversant la muqueuse grâce à des cellules épithéliales modifiées, appelées cellules M [11]. Les lymphocytes B activés sont ensuite engagés dans un cycle de maturation de 6 jours dans le sang circulant, aboutissant à une migration vers des zones dispersées dans la muqueuse, généralement à proximité de la formation lymphoïde d'origine [10]. Les lymphocytes B se différencient alors en plasmocytes et sécrètent des IgA. Ce système constitue le modèle de référence pour la mise au point des protocoles vaccinaux par voie muqueuse. Il présente cependant des inconvénients majeurs : le premier est d'ordre opérationnel, et est lié à la difficulté d'induction des anticorps S-IgA chez

\footnotetext{
$\mathrm{m} / \mathrm{s} n^{\circ} 12$, vol. 15 , décembre 99
}

l'homme. Le second est d'ordre théorique, et concerne le retard d'apparition de cette réponse immunitaire, qui peut atteindre 3 semaines, ainsi que l'absence fréquente de mémoire immunitaire, ces deux limitations étant incompatibles avec une fonction de première barrière qui nécessite la présence de l'anticorps à l'arrivée de l'agent infectieux.

Ces difficultés conceptuelles ont été récemment levées par la mise en évidence de nombreux anticorps naturels dans les sécrétions humaines [12]. Ces anticorps, décrits par l'équipe de S. Avraméas [13, 14], existent avant toute stimulation antigénique. Ce sont des molécules plurispécifiques, chacune capable de reconnaître plusieurs épitopes non apparentés, principalement autoantigéniques. La structure des régions hypervariables de ces anticorps est généralement peu modifiée par rapport à leur structure germinale $[15,16]$, et une théorie récente suggère que ces anticorps correspon- dent à une forme moderne de molécules ancestrales impliquées dans la clairance des composants du soi [17]. Les anticorps naturels réagissent également avec de nombreux constituants microbiens, fournissant ainsi une défense «immédiate» existant avant tout contact préliminaire avec l'antigène microbien. Cette défense est cependant limitée par l'existence d'antigènes (toxines ou addressines) impliqués dans la pathogénicité des agents les plus infectieux mais mal reconnus par les anticorps naturels. Les anticorps S-IgA induits par l'antigène sont sécrétés par des cellules $B$ de type B2, originaires du MALT, tandis que les anticorps naturels SIgA sont généralement sécrétés par des cellules de type B1, autorenouvelées dans la muqueuse où elles constituent plus de $50 \%$ des cellules B intestinales chez la souris [18].

\section{Anticorps d'origine sérique, immunité passive et anticorps locaux}

Les anticorps présents dans les sécrétions possèdent différentes activités physiologiques selon leur isotype, leur mode d'induction et leur origine (Tableau I). En dehors de la transcytose, des anticorps peuvent diffuser du sang vers les sécrétions lorsque la perméabilité de la muqueuse est augmentée en raison d'une inflammation locale ou d'une simple dilatation. Le foie est également le siège d'une libération importante d'IgG sériques dans le tube digestif. Lors de leur catabolisme hépatique, les IgG du sérum sont éliminées dans les voies biliaires, puis vers l'intestin, où elles sont ensuite dégradées en fragments Fab après clivage par les protéases endogènes [4]. Les IgA et IgM sériques sont également relarguées, mais ce phénomène est physiologiquement négligeable, car l'affinité de leurs fragments Fab est beaucoup plus faible. Chez les rongeurs (rats, souris, cobayes) et les lagomorphes (lapins, lièvres), le statut polymérique des IgA sériques et la présence du récepteur poly-Ig récepteur sur la membrane des hépatocytes entraînent la formation d'une "pompe hépatique », libérant par transcytose locale des S-IgA dérivées du sérum, mais indiscernables des S-IgA muqueuses [19]. 
Tableau I

PROPRIÉTÉS DES PRINCIPAUX ANTICORPS DES SÉCRÉTIONS

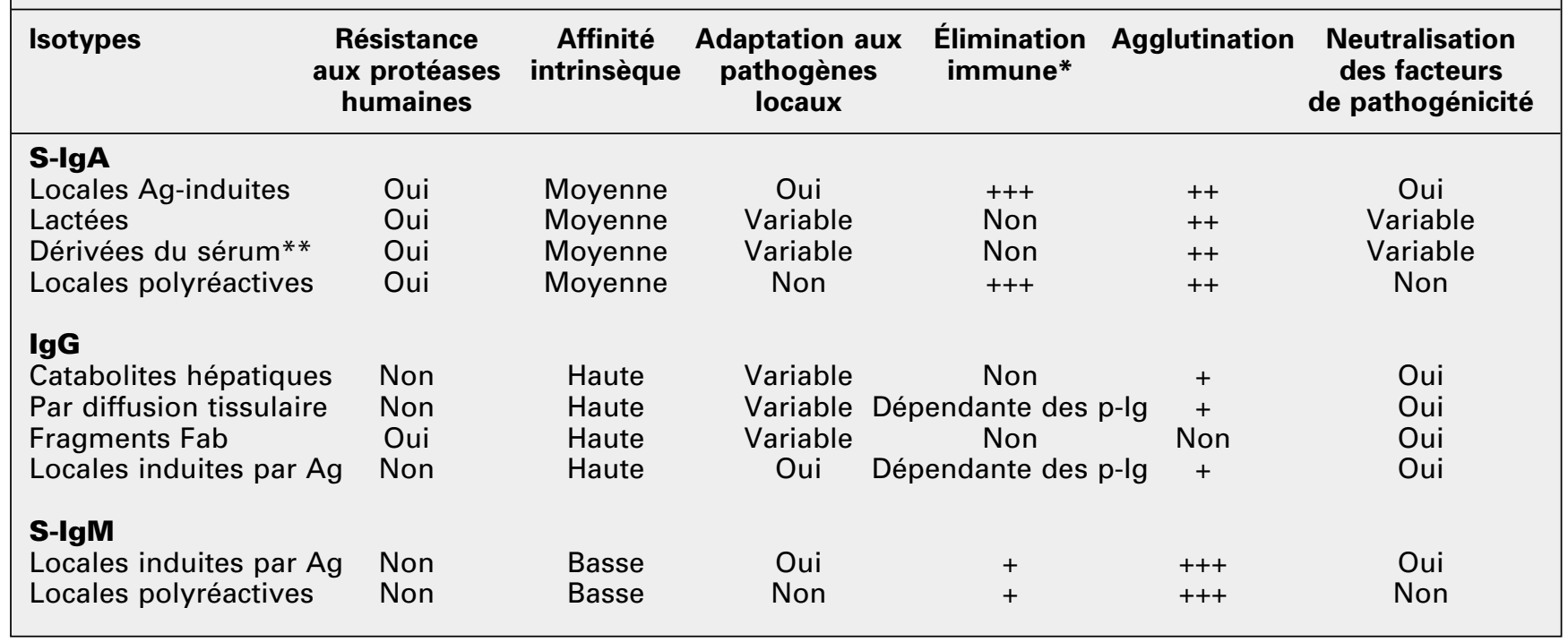

* Durant la transcytose des IgA et des IgM polymériques (p-lg). ** Chez les rongeurs et les lagomorphes. Ag: antigène.

L'induction par voie parentérale d'anticorps S-IgA d'origine sérique est donc possible chez les principales espèces de laboratoire et ne doit donc pas être confondue avec une immunisation muqueuse. L'activité protectrice de ces anticorps est d'ailleurs limitée à l'exclusion immune. Cette différence avec la protection apportée par des S-IgA produites localement est apparue dans des travaux récents de vaccination par voie orale ou parentérale contre Salmonella typhimurium chez la souris [20]. De la même manière, les $\mathrm{S}$-IgA du lait maternel induisent chez le nourrisson une immunité passive, également limitée à l'exclusion immune. A l'inverse, la découverte récente d'une réponse muqueuse locale à anticorps IgG chez l'homme [21] ouvre de nouvelles voies pour la vaccination muqueuse. Il semble que dans certaines conditions expérimentales, il soit possible d'induire dans les sécrétions des anticorps IgG de haute affinité dont des antitoxines tétaniques [22].

\section{Les agents inhibiteurs ou amplificateurs: protéases et protéine Fv}

Bien que la colonisation microbienne stimule la synthèse d'anticorps et s'oppose au développement de nom- breux germes pathogènes, elle peut également interférer avec les fonctions effectrices des anticorps dans les sécrétions. Ainsi, les S-IgA qui résistent aux protéases endogènes peuvent cependant être dégradées par des IgA-protéases bactériennes généralement dirigées contre la sous-classe IgA1 [23]. Par ailleurs, certains des composants de la flore, ainsi que des virus hépatotropes, peuvent induire la libération dans l'intestin d'un co-récepteur des SIgA, la protéine $\mathrm{Fv}$ [24], qui se fixe spécifiquement sur le fragment variable $(\mathrm{Fv})$ (mais à distance du site anticorps) des immunoglobulines de la famille VH3 [25]. Cette sialoprotéine de $175 \mathrm{kDa}$, résistante aux protéases, porte 6 sites de liaison, entraînant la formation de larges complexes macromoléculaires avec les S-IgA et leurs fragments $\mathrm{F}(\mathrm{ab}) 2$ (figure 3 ). Dans les selles, ces complexes, appelés «forteresses immunitaires», peuvent atteindre $3000 \mathrm{kDa}$ [26] ; ils potentialisent l'activité effectrice des S-IgA et de leurs fragments, notamment l'agglutination, et sont particulièrement intéressants pour potentialiser l'action des anticorps naturels [12].

\section{La conception d'ensemble}

La multiplicité des mécanismes impliqués dans l'immunité humorale des sécrétions (Tableau I) reflète l'importance de leur rôle protecteur anti-infectieux. Ces mécanismes correspondent à des perfectionnements rendus progressivement indispensables au cours de l'évolution, notamment par le passage à l'homéothermie, qui a nécessité de «soulager»le système macrophagique, jusqu'alors suffisant pour les animaux à sang froid. Certains outils nécessaires à l'immunité humorale des muqueuses sont cependant très anciens. Ainsi, la chaîne J, nécessaire à la fixation sur le récepteur $\mathrm{SC}$, était déjà présente chez la plupart des mollusques [27] alors que ces animaux ne possèdent pas d'immunoglobulines. De même, l'IgM est apparue chez les poissons cartilagineux et le récepteur SC a été détecté chez les amphibiens primitifs [28], tandis que les immunoglobulines de poissons sont formées essentiellement d'anticorps naturels polyréactifs [29]. L'immunité muqueuse relayée par des S-IgM locales polyréactives est donc probablement apparue la première au cours de la phylogenèse. Chez les mammifères, le remplacement des S-IgM par les SIgA a représenté une amélioration considérable du rendement de la transcytose [30] et de la persistance de ces polymères dans le tube digestif. Ces anticorps naturels ont fourni 


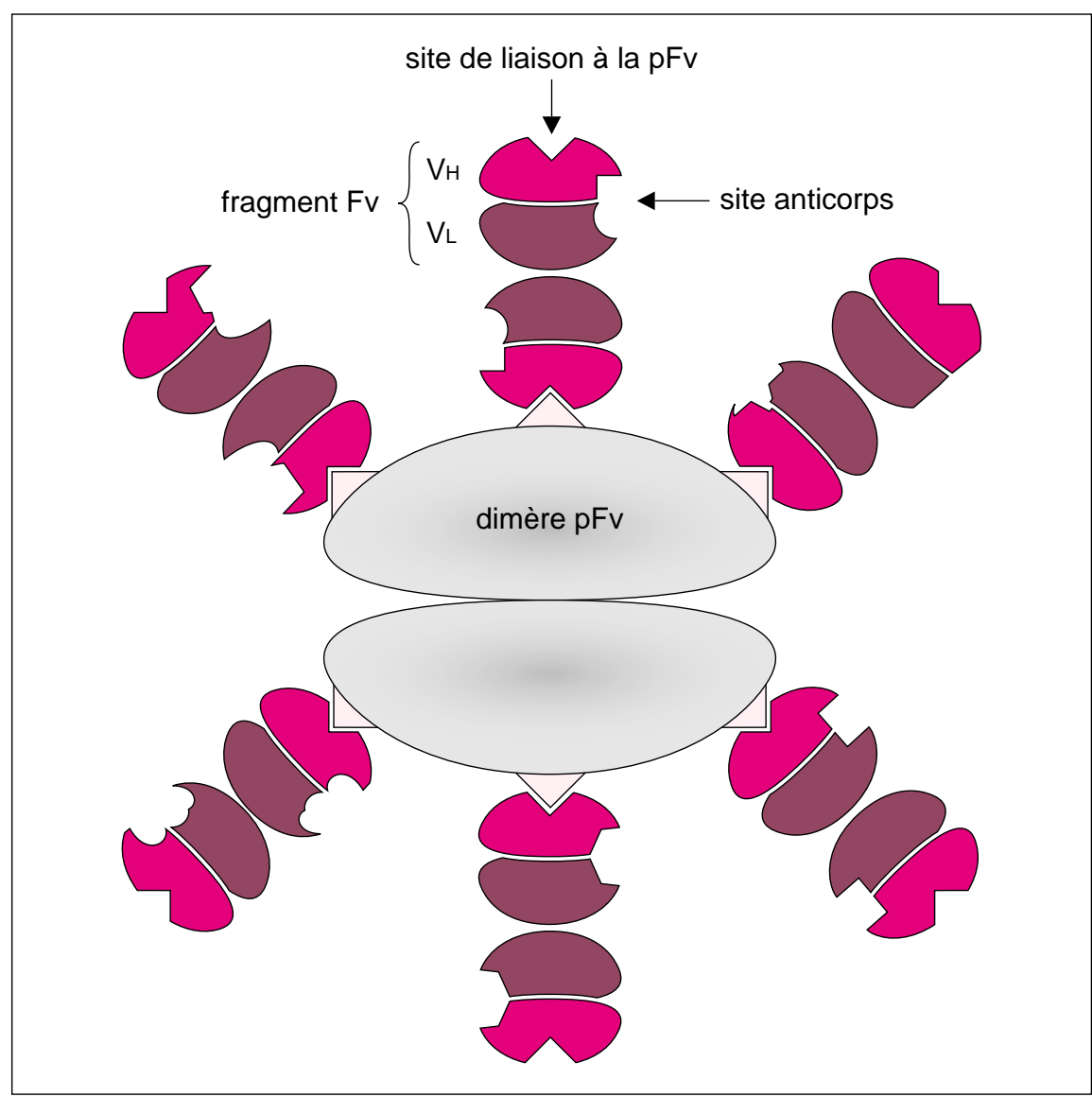

Figure 3. Structure d'une "petite" forteresse immune (800 kDa) formée par la protéine $F v$ (au centre) et 6 fragments $F\left(a b^{\prime}\right) 2 \alpha$. La molécule est formée de deux sous unités de $~ 82 \mathrm{kDa}$ qui fixent les domaines VH appartenant à la famille VH3, sans inhiber le site anticorps. L'ensemble formé d'un agrégat d'anticorps polyclonaux permet d'amplifier ou de rétablir les propriétés des $S$-IgA liées à leur caractère polymérique. Celui-ci potentialise leurs propriétés agglutinantes et facilite l'exclusion immune.

dès leur apparition un moyen de défense simple et suffisamment efficace pour maintenir un bon équilibre avec la flore commensale et des inoculums peu abondants ou peu pathogènes. Chez les rongeurs, la transcytose hépatique des IgA chez les rongeurs ainsi que la diffusion des IgG sériques et leur catabolisme hépatique ont apporté l'aide d'anticorps induits par l'antigène. Ces anticorps persistent après disparition de l'agent pathogène et augmentent rapidement lors d'un nouveau contact. Ils présentent cependant l'inconvénient d'être adaptés à la pathologie générale et non aux infections locales, d'où l'intérêt des derniers-venus parmi les anticorps muqueux: les IgG et S-IgA locales induites par l'antigène. Ces anticorps sont adaptés aux pathogènes locaux, spécificites tres variables d' faciles à induire et ont une meilleure affinité. Ils ont des propriétés neutralisantes vis-à-vis des virus et des toxines, même après clivage enzymatique. Il semble donc que la complémentarité des anticorps de ces deux isotypes (S-IgA et $\operatorname{IgG}$ ), induits par l'antigène et synthétisés localement, soit d'un grand intérêt dans l'immunité muqueuse.

L'analyse récente des différents aspects de l'immunité sécrétoire relayée par les anticorps éclaircit considérablement notre compréhension de ce système immunitaire d'importance majeure sur les plans quantitatif et fonctionnel. Ces nouvelles conceptions d'ensemble permettent de suggérer les raisons probables de l'échec de certains vaccins et élargissent le champ de recherche des modes d'induction et d'analyse de la réponse muqueuse, facteur-clé du développement des vaccins locaux

\section{RÉFÉRENCES}

1. Botor C, Couëdel-Courteille A, Venet A, Guillet JG. Immunité locale et vaccination. Med Sci 1995; 11 : 703-11.

2. Cerf-Bensussan N, Guy-Grand D. Intestinal intraepithelial lymphocytes. Gastroenterol Clin North Am 1991; 20 : 549-76.

3. Hocini H, Barra A, Bélec L, Iscaki S, Preud'homme JL, Bouvet JP. Systemic and secretory humoral immunity in the normal vaginal tract. Scand J Immunol 1995; 42: 26974 .

4. Quan CP, Ruffet E, Arihiro K, Pirès R, Bouvet JP. High affinity serum-derived Fab fragments as another source of antibodies in the gut lumen of both neonates and adults. Scand J Immunol 1996; 44: 108-14. spécificités très variables d'une muqueuse à l'autre. Les avantages respectifs des deux isotypes dépendent des conditions locales. Les S-IgA prédominent dans la salive, le lait et le tube digestif, probablement en raison de leur résistance aux protéases endogènes en aval, et de leur fixation supérieure à celle des Faby sur la protéine Fv pour former des forteresses hyperagglutinantes. Elles sont également indispensables à l'élimination immune, et leur caractère agglutinant dû à la polymérisation facilite leur entraînement par le mucus sur une longue distance. A l'inverse, les IgG prédominent dans les sécrétions génitales qui ne contiennent pas de protéases endogènes, mais des IgA1 protéases microbiennes [3]. Les anticorps IgG sont probablement plus
5. Stokes CR, Southill JF, Turner MW. Immune exclusion is a function of IgA. Nature 1975; 255: 745-6.

6. Kaetzel CS, Robinson JK, Chantalacharuvu KR, Vareman JP, Lamm ME. The polymeric immunoglobulin receptor (secretory component) mediates transport of immune complexes across epithelial cells : a local defense function for IgA. Proc Natl Acad Sci USA 1991; 88: 8796-800.

7. Mazanec MB, Kaetzel CS, Lamm ME, Fletcher D, Nedrud JG. Intracellular neutralization of virus by immunoglobulin A antibodies. Proc Natl Acad Sci USA 1992; 89: 6901-5.

8. Capron A, Dessiaut JP, Capron M, Ouma $\mathrm{JH}$, Butterworth A. Immunity to schistosomes; progress toward vaccine. Science 1987 ; 238: 1065-72.

9. Besredka A. Local immunization. Baltimore : Williams and Wilkins, 1927. 


\section{RÉFÉRENCES}

10. Brandtzaeg P, Farstad IN. The human mucosal B-cell system. In: Ogra PL, Mestecky J, Lamm ME, Strober W, Bienenstock J, McGhee JR, eds. Mucosal immunology. San Diego: Academic Press, 1999: 439-68.

11. Neutra MR, Pringault E, Kraehenbuhl JP. Antigen sampling across epithelial barriers and induction of mucosal immune responses. Annu Rev Immunol 1996; 14: 275300

12. Quan CP, Berneman A, Pirès R, Avrameas S, Bouvet JP. Natural secretory immunoglobulin A polyreactive autoantibodies as a possible immune barrier in humans. Infect Immun 1997; 69: 3997-4004.

13. Guilbert B, Dighiero G, Avrameas S. Naturally occurring antibodies against nine common antigens. I. Detection, isolation and characterization. J Immunol 1982; 128 : 2779-87.

14. Dighiero G, Guilbert B, Avrameas S. Naturally occurring antibodies against nine common antigens in human sera II. High incidence of of monoclonal Ig exhibiting antibody activity against actin and tubulin, and sharing antibody specificities with natural antibodies. J Immunol 1982; 28: 2788-92.

15. Baccala R, Quang TV, Gilbert M, Ternynck T, Avrameas S. Two murine natural autoantibodies are encoded by nonmutated germ-line genes. Proc Natl Acad Sci USA 1989 ; 86: 4624-8.

16. Diaw L, Magnac C, Pritsch O, Buckle M, Alzari P, Dighiero G. Structure and affinity studies of IgM polyreactive natural autoantibodies. J Immunol 1997; 158: 968-76.

17. Bouvet JP, Dighiero G. From natural polyreactive autoantibodies to à la carte monoreactive antibodies to infectious agents: is it a small world after all? Infect Immun $1998 ; 66: 1-4$.

18. Murakami M, Honjo T. Involvement of B-1 cells in mucosal immunity and autoimmunity. Immunol Today 1995; 16: 534-8.

19. Delacroix DL, Hodgson HJF, McPherson A, Dive C, Vaerman JP. Selective transport of polymeric IgA in bile. Quantitative relationship of monomeric and polymeric IgA, IgM and other proteins in serum, bile and saliva. J Clin Invest 1982; 70 : 230-41.

20. Allaoui-Attarki K, Pecquet S, Fattal E, et $a l$. Protective immunity against Salmonella typhimurium elicited in mice by oral vaccination with phosphorycholine encapsulated in poly (D,L-lactide-co-glycolide) microspheres. Infect Immun 1997; 65 : 853-7.

21. Berneman A, Bélec B, Fischetti VA, Bouvet JP. The specificity patterns of human immunoglobulin $\mathrm{G}$ antibodies in serum differ from those in autologous secretions. Infect Immun 1998; 66: 4163-8.

22. Bouvet JP, Bélec L, Pirès R, Pillot J. Immunoglobulin $\mathrm{G}$ antibodies in human vaginal secretions after parenteral vaccination. Infect Immun 1994; 62: 3957-61.

23. Kilian M, Reinholdt J, Lomholt H, Poulsen K, Frandsen EVG. Biological significance of IgAl proteases in bacterial colonization and pathogenesis : critical evaluation of experimental evidence. Acta Pathol Microbiol Immunol Scand 1996; 104: 321-38.

24. Bouvet JP, Pirès R, Lunel-Fabiani $\mathrm{F}$, et al. Protein $\mathrm{F}$ : a novel $\mathrm{F}(\mathrm{ab})$ binding factor, present in normal liver, and largely released in the digestive tract during hepatitis. J Immunol 1990 ; 145 : 1176-80.

25. Silverman GJ, Pirès R, Bouvet JP. An endogenous sialoproteinand a bacterial $\mathrm{B}$ cell superantigen compete in their $\mathrm{VH}$ family-specific binding interactions with human Igs. J Immunol 1996; 157: 496-502.

26. Bouvet JP, Pirès R, Iscaki S, Pillot J. Nonimmune macromolecular complexes of Ig in human gut lumen: probable enhancement of antibody functions. J Immunol 1993; 151 : 2562-71.

27. Takahashi $\mathrm{T}$, Iwase $\mathrm{T}$, Takenouchi $\mathrm{N}$, et al. The joining (J) chain is present in invertebrates that do not express immunoglobulins. Proc Natl Acad Sci USA 1996; 93: 1886-91.

28. Fellah JS, Iscaki S, Vaerman JP, Charlemagne J. Transient developmental expression of IgY and secretory component like protein in the gut of the axolotl (Ambystoma mexicanum). Dev Immunol 1992; 2: 181-90.

29. Gonzalez R, Charlemagne J, Mahana W, Avrameas S. Specificty of natural serum antibodies present in phylogenetically distinct fish species. Immunology 1988; 63: 31-6.

30. Natvig IB, Johansen FE, Nordeng TW, Haraldsen G, Brandtzaeg P. Mechanism of enhanced external transfer of dimer IgA over pentameric IgM: studies of diffusion, binding to the human polymeric Ig receptor, and epithelial transcytosis. I Immunol $1997 ; 159: 4330-40$.

\section{Summary}

Complexity of the first immune barrier against mucosal pathogens

Antibody-dependent mucosal immunity consists of different and complementary mechanisms which emerged during evolution. It is now established that S-IgA is not the only isotype of Ig found in secretions, and that antigen-induced and naturally occurring antibodies play different roles. The antigen-induction of S-IgA antibodies by classical methods is inefficient, which may explain the persistent failures of mucosal vaccines. Polyreactive natural antibodies, which precede any preliminary contact with antigens, appear to be the major agents in the first mucosal defence barrier against pathogens. The presence of high affinity Fab fragments in the digestive tract, originating from liver release of serum IgG, as well as the evidence for a regionalization of mucosal IgG have led to reconsider the classical concepts in mucosal immunity. The major implications of this recent advances will be to open new fields of investigations for trials of future mucosal vaccines.

\section{Remerciements}

Je remercie Sylvio Iscaki pour ses fructueuses critiques de ce manuscrit et Pierre Kitmacher pour la réalisation des figures.

\section{TIRÉS À PART}

J.P. Bouvet.

\section{GERDA}

Groupe d'Études et de Recherches en Dermato-Allergologie PARIS - Palais des Congrès - 5 au 7 octobre 2000

Dermato-allergo-pédiatrie - Pathologies allergiques des muqueuses

Organisation scientifique :

Dr Annik Pons-Guiraud/10, bd Malesherbes, 75008 Paris, France.

Tél. : 01426632 01/Fax: 014266 '32 13-E-mail : Annick.Pons-guiraud@wanadoo.fr

Organisation technique :

MELTHEM/95, rue de Lourmel, 75015 Paris, France.

Tél. : 01442616 48/Fax: 0145543621 - E-mail : melthem@club-internet.fr 